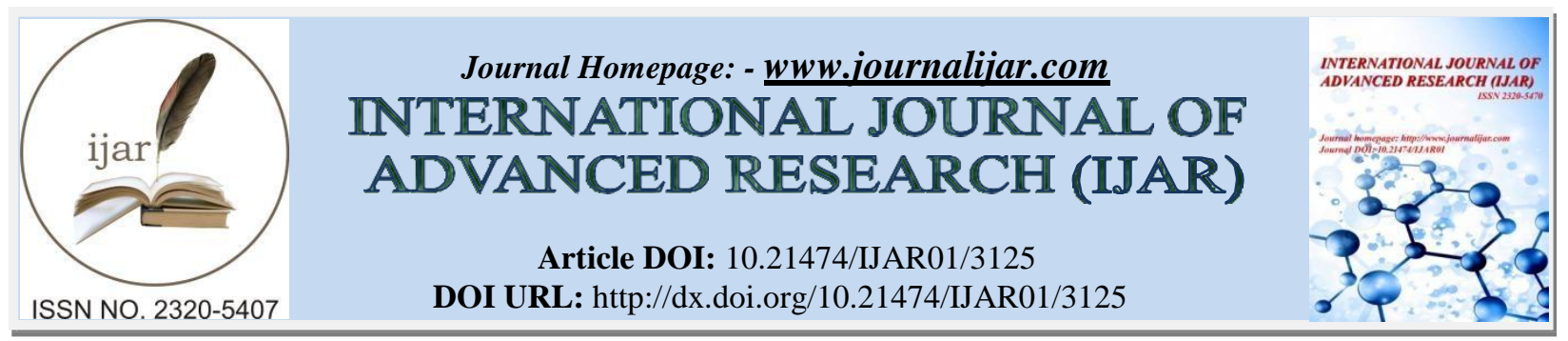

RESEARCH ARTICLE

\title{
THE IMPORTANCE OF CLINICOPATHOLOGICAL FEATURES FOR DIFFERENTIATION BETWEEN CROHN'S DISEASE AND ULCERATIVE COLITIS.
}

\author{
Ghada E. Esheba ${ }^{1}$, Ghadeer F. Alharthi ${ }^{2}$, Duaa A. Alhejaili ${ }^{2}$, Rawan E. Hudairy ${ }^{2}$, Wafaa A. Altaezi ${ }^{2}$, Raghad \\ M. Alhejaili ${ }^{3}$. \\ 1. Associate Professor of Pathology; Department of Pathology, Faculty of Medicine, Tanta University,Egypt. \\ 2. Medical intern, Department of Pathology, Faculty of Medicine, Umm Al-Qura University,KSA. \\ 3. $5^{\text {th }}$ year Medical student, Umm Al-Qura University.
}

Manuscript Info

Manuscript History

Received: 08 December 2016

Final Accepted: 05 January 2017

Published: February 2017

\section{Abstract}

Background: Inflammatory bowel disease (IBD) consists of two specific gastrointestinal disorders: ulcerative colitis (UC) and Crohn's disease (CD). Despite their distinct natures, these two diseases share many similar etiologic, clinical and pathological features, as a result, their accurate differential diagnosis may sometimes be difficult. Correct diagnosis is important because surgical treatment and long-term prognosis differ from UC and CD.

Aim: This study aims to study the characteristic clinicopathological features which help in the differential diagnosis between UC and CD, and assess the disease activity in ulcerative colitis.

Materials and methods: This study was carried out on 50 selected cases, referred to the department of pathology, AlnoorSpecialist Hospital. The cases included 27 cases of UC and 23 cases of CD. All the cases were examined using H\&E and immunohistochemically for bcl-2expression.

Results: Characteristic features of UC include: decrease in mucous content, irregular villous surface, crypt distortion, and cryptitis, whereas the main cardinal histopathological features seen in $\mathrm{CD}$ were: epitheloid granuloma, transmural chronic inflammation, absence of mucin depletion, irregular surface, or crypt distortion. 3 cases of UC were found to be associated with dysplasia. UC mucosa contains fewer Bcl-2+ cells compared with CD mucosa.

Conclusion: This study using multiple parameters such clinicopathological features and $\mathrm{Bcl}-2$ expression as studied by immunohistochemical stain, helped to gain an accurate differentiation between UC and CD. Furthermore, this work spotted the light on the activity and different grades of UC which could be important for the prediction of relapse.

Copy Right, IJAR, 2017,. All rights reserved.

\section{Introduction:-}

Inflammatory bowel disease is an idiopathic disease caused by an immune response. Two types of IBD are present, ulcerative colitis (UC) and Crohn's disease (CD). To establish a diagnosis of IBD, exclusion of other causes of 
inflammation will be done first and then the signs and symptoms, blood tests, radiological studies, macroscopic and microscopic examination of biopsies will be used to differentiate between CD and UC (1).

The intestinal inflammatory processes in IBD are triggered by genetic susceptibly in a patient who has environmental factors, defect in the intestinal epithelial tight junction barrier function, and dysfunction of innate and adaptive response pathways, especially the $\mathrm{T}$ cell response. The adaptive immune response in $\mathrm{CD}$ has long been considered to be driven by the response of Th1 cells. On the other hand, UC has been associated with an unconventional Th2 cells response. Furthermore, Th17 cells have been involved in the gut inflammatory response in $\operatorname{IBD}(2,3)$.

The common presenting complain of IBD is chronic diarrhea with or without blood or mucus. Bloody stool, is more typical of UC. Other symptoms are abdominal pain, weight loss, fever, fatigue and sweat. Most common extraintestinal symptoms are peripheral arthritis, aphthous ulcer, ankylosing spondylitis and erythema nodosum $(4,5)$.

Ulcerative colitis may lead to one or more of the complications such as fulminant colitis, severe bleeding, toxic megacolon, perforation and an increased risk for the development of malignancy. Perianal diseases such as fistulae, skin tags, or anal fissures are complications of crohn's disease occur in $35 \%$ of patients $(5,6)$.

The use of serological markers such as antibodies to Saccharomyces cerevisiae may help in differentiating UC from CD; (ASCA) is present in $40 \%$ to $80 \%$ of patients with CD while the anti-neutrophil cytoplasmic antibody (pANCA) is detected in nearly $75 \%$ of patients with $\mathrm{UC}(7,8)$.

In an abdominal plain film, a narrowed terminal ileum (kantor's string sign) is a characteristic finding of CD in upper GI with small bowel follow-through, while in UC, it is important to evaluate colonic distention to rule out toxic megacolon. Barium enema demonstrates lead pipe appearance that is an indication of chronic UC characterized by loss of haustrations and shortening \& narrowing of colon. Abscess formation and fistula on abdominal computed tomography are associated with $\mathrm{CD}(9)$.

CD may affect any area within the gastrointestinal tract while UC affects the rectum and extends to the colon in a continuous fashion. The endoscopic features in $\mathrm{CD}$ are characterized by fat wrapping, thickening of the intestinal wall, ileum involvement and other features such as skip lesions, cobblestoning, confluent deep linear ulcers, aphthoid ulcers, deep fissures, fistulas and strictures. On the other hand, the endoscopic features in UC are not characteristics but mainly include erythema, mucosal vascular congestion, loss of apparent vascular pattern in mild inflammation and clear demarcation between inflamed and normal areas. Granularity, mucosal erosions and mucosal friability can be seen in advanced cases. While Spontaneous bleeding and ulceration located in the inflamed mucosa will be present in severe cases $(10,11)$.

For estimating the histological activity of ulcerative colitis, Geboesscore is used. (Table 1) (12).

The Management of IBD depends on the severity of disease (4). Medications include, steroids, immunosuppressive agents, aminosalicylates and anti-inflammatory drugs (7). Mild cases are treated by anti-inflammatory drugs orallyor as enemas. Severe cases can be treated with immunomodulatoror immunosuppressant medications either orally, by an IV infusion, or a subcutaneous injection (4). Infliximab is used for moderate to severe CD and UC for preservation of remission, also when managed by immunomodulators and mesalamine is failed.(13).

Surgical management have a role in improving quality of life but still there is probability of recurrence the disease after surgery (5). Surgical options are indicated if medical treatment failedor in UC or CD associated with neoplasia(14). Also, if there is any complication such as: perforation, abscess, malnutrition and intestinal obstruction.(5).

IBD's patients have a higher risk for developing colon cancer later on (6). Patients with UC have higher mortality rate than those with CD.(15).

Table 1:-

\begin{tabular}{|c|l|l|l|}
\hline \multicolumn{2}{|l|}{ Grade 0: structural changes } & \multicolumn{2}{l|}{ Grade 3: neutrophils in epithelium } \\
\hline Subgrades & & Subgrades & \\
\hline 0.0 & No changes & 3.0 & None \\
\hline 0.1 & Mild abnormality & 3.1 & $<5 \%$ of crypts \\
\hline
\end{tabular}




\begin{tabular}{|c|c|c|c|c|c|}
\hline 0.2 & \multicolumn{3}{|c|}{ Mild to moderate diffuse or multifocal irregularity } & 3.2 & $<50 \%$ of crypts \\
\hline 0.3 & \multicolumn{3}{|c|}{ Severe diffuse or multifocal abnormalities } & 3.3 & $>50 \%$ of crypts \\
\hline \multicolumn{4}{|c|}{ Grade 1: chronic inflammatory infiltrate } & \multicolumn{2}{|c|}{ Grade 4: crypt destruction } \\
\hline 1.0 & \multicolumn{3}{|c|}{ No progression } & 4.0 & None \\
\hline 1.1 & \multicolumn{3}{|l|}{ Mild progression } & 4.1 & neutrophils in part of the crypt \\
\hline 1.2 & \multicolumn{3}{|c|}{ Moderate progression } & 4.2 & marked depletion \\
\hline 1.3 & \multicolumn{3}{|c|}{ Marked progress } & 4.3 & absolute crypt loss \\
\hline \multicolumn{4}{|c|}{ Grade 2: lamina propria neutrophils and eosinophils } & \multicolumn{2}{|c|}{ Grade 5: erosion and ulceration } \\
\hline \multicolumn{2}{|c|}{ 2A: eosinophils } & \multicolumn{2}{|c|}{ 2B: neutrophils } & 5.0 & none \\
\hline $2 \mathrm{~A} .0$ & No increase & $2 \mathrm{~B} .0$ & None & 5.1 & $\begin{array}{l}\text { Recovering epithelium + adjacent } \\
\text { inflammation }\end{array}$ \\
\hline 2A.1 & Mild increase & 2B.1 & Mild increase & 5.2 & Probable erosion, focally stripped \\
\hline 2 A. 2 & Moderate increase & 2B. 2 & Moderate increase & 5.3 & Unequivocal erosion \\
\hline $2 \mathrm{~A} .3$ & Marked increase & 2B. 3 & Marked increase & 5.4 & Ulcer or granulation tissue \\
\hline
\end{tabular}

\section{Materials and methods:-}

This study was carried out on 50 selected cases, referred to the department of pathology, Alnoor Specialist Hospital during the period between 2010-2015. In all cases, the diagnosis was made from the clinical history, endoscopy, and radiological features which were obtained from the accompanying clinical sheets, and confirmed by microscopic examination.

The collected cases were sent as colonoscopy specimens (41 cases) or surgically removed by colectomy ( 9 cases). The cases include 27 cases of UC and 23 cases of CD.

\section{Methods:-}

For every case, the following data were recorded: age, presenting symptoms, site of involvement, gross pathology, and microscopic picture.

The specimens were fixed in 10\% formol saline and embedded in paraffin. The paraffin blocks were serially sectioned at 4-5 microns and stained by:

1. Hematoxylin and Eosin (H\&E).

2. Immunohistochemical staining of Bcl-2: Four $\mu \mathrm{m}$ thick sections were cut from routine paraffin embedded blocks then deparaffinized in xylene, and hydrated in graded alcohols. Immunostaining was performed with the Dakoautostainer. The positive cells (mucosal $\mathrm{T}$ cells) were approximately measured and expressed as mild, moderate, or markedexpression.

\section{Statistical analysis:-}

Data were analyzed using SPSS version 12.0.0 for Windows (SPSS Inc., Chicago, IL). Scale variables were presented as the mean \pm standard deviation (mean $\pm \mathrm{SD}$ ) or percentages as appropriate.

\section{Results:-}

\section{Clinical characteristic:-}

The current study included 27(54\%) cases of UC and 23(46\%) cases of CD.

In UC, 15 cases (55.6\%) were females while the remaining 12 cases $(44.4 \%)$ were males.

The age and sex of the patients were summarize in table 2 .

The differences in age and sex between UC and DC were not significant.

Table 3:-

\begin{tabular}{|l|l|l|l|}
\hline Disease & Age range & Mean age & Sex \\
\hline Ulcerative colitis & $23-59$ & $38.84 \pm 14.26$ & Female 15 (55.6\%) \\
\cline { 3 - 4 } & & & Male 12 $(44.4 \%)$ \\
\hline Crohn's disease & $20-60$ & $35.25 \pm 7.93$ & Female 10 (43.5\%) \\
\cline { 3 - 3 } & & & Male 13 $(56.5 \%)$ \\
\hline
\end{tabular}


The main clinical manifestations in UC were diarrhea, rectal bleeding, abdominal pain, and some patients complained of extraintestinal symptoms such as arthritis as well. On the other hand, patients with CD were mainly presented by diarrhea, abdominal pain and anal complications such as fissures.

Colonoscopic examination in UC revealed multiple pleomorphic ulcers in 11 (40.7\%) cases with evident diffuse or focal erythema. Mucopurulent exudate was present in $6(22.2 \%)$ cases. The mucosa was atrophic and no ulcers could be detected in the remaining cases. Pseudopolyposis was detected in $2(7.4 \%)$ cases. While in CD, colonoscopic examination showed erythematous plaques and discrete ulcers (I-5 mm in diameter) in 11(47.8\%) cases. Evident skip lesions were detected in 9 (39.1\%) cases, while rectal sparing was found in all examinedcases.

\section{Histopathological study:-}

\section{Ulcerative colitis}

The different grades of UC were summarized in Table 3 and were shown in Fig.2.

Table 3:- Different grades of UC in this study.

\begin{tabular}{|l|l|}
\hline Grades & Number of cases \\
\hline 0.3 & $1(3.70 \%)$ \\
\hline 1.3 & $2(7.41 \%)$ \\
\hline $2 \mathrm{~A} .2$ & $2(7.41 \%)$ \\
\hline $2 \mathrm{~B} .2$ & $2(7.41 \%)$ \\
\hline $2 \mathrm{~B} .3$ & $1(3.70 \%)$ \\
\hline 3.1 & $2(7.41 \%)$ \\
\hline 3.3 & $4(14.81 \%)$ \\
\hline 4.1 & $3(11.11 \%)$ \\
\hline 4.3 & $4(14.81 \%)$ \\
\hline 5.4 & $3(11.11 \%)$ \\
\hline Low grade dysplasia & $2(7.41 \%)$ \\
\hline High grade dysplasia & $1(3.70 \%)$ \\
\hline
\end{tabular}

In grade 0.3 , we found severe abnormalities in the crypts structure, including crypt branching, crypt distortion and villous appearance.

In grade 1.3, we found marked increase of chronic inflammatory cells infiltration in the mucosa.

In grade $2 \mathrm{~A} .2$ of $\mathrm{UC}$, we found moderate increase of eosinophils in lamina propria. While in grade 2B.2, there was moderate increase in neutrophils. And in 2B.3 it was a marked increase.

In grade 3.1 of UC, we found that less than $5 \%$ of the crypts to be infiltrated by neutrophils. While, in grade 3.3, we found that more than $50 \%$ of crypts were infiltrated.

In grade 4.1 of UC, there was excess of neutrophils in parts of the crypts and the crypts showed a severe form of structural abnormalities. And in grade 4.3, there was prominent crypt atrophy. Also, multiple crypt abscesses were found which were formed of acinar structure containing neutrophils and pus cells.

In grade 5.4 of UC, we found multiple superficial mucosal ulcers.

In this study 2 (7.4\%) cases showed low-grade dysplasia and one (3.7\%) case showed high grade dysplasia, the histopathological features seen in low-grade dysplasia were: nuclear hyperchromasia associated with pleomorphism and Mucin vacuoles were distributed and located in the basal part of cytoplasm which called "dystrophic goblet cells".

In high grade dysplasia, the following features were seen: enlarged hyperchromatic nuclei, marked nuclear stratification with crowding, loss of nuclear polarity, marked architectural disarray and decreased mucin production.

\section{Immunohistochemical study:-}

Examination of sections stained immunohistochemically for the antiapoptotic Bcl-2 protein revealed mild expression of the $\mathrm{Bcl}-2$ protein and this indicate that $\mathrm{UC}$ mucosa contains few number of $\mathrm{Bcl}-2+$ cells. 


\section{Crohn's disease:-}

All cases in this work showed transmural inflammation in the form of chronic inflammatory infiltrate (lymphocytes, macrophages, plasma cells and giant cells) affecting all the layers up to the serosa.

Non caseating granuloma (submcosal and subserosa) formed of epitheloid cells and few giant cells were seen in 14 (60.9\%) cases. Dilatation of lymphatic channels was detected in $5(21.7 \%)$ cases. Fissuring ulcer was present in 2 (8.7\%) cases. Focal crypt abscess was seen in 3(13\%) cases. Transmural lymphoid aggregates were present in $2(8.7 \%)$ cases. Submucosal nerve fiber hyperplasia was evident in $4(17.4 \%)$ cases. $2(8.7 \%)$ cases exhibited architectural alteration with patchy neutrophils, mucin depletion and damage in the crypt epithelium. Fig. (3)

\section{Immunohistochemical study:-}

Examination of sections stained immunohistochemically for the antiapoptotic Bcl-2 protein revealed marked expression of the $\mathrm{Bcl}-2$ protein and this indicate that in $\mathrm{CD}$ there is a defect in apoptosis which in turn lead to prolongation of $\mathrm{T}$ cell survival and contribute to their accumulation in the mucosa.
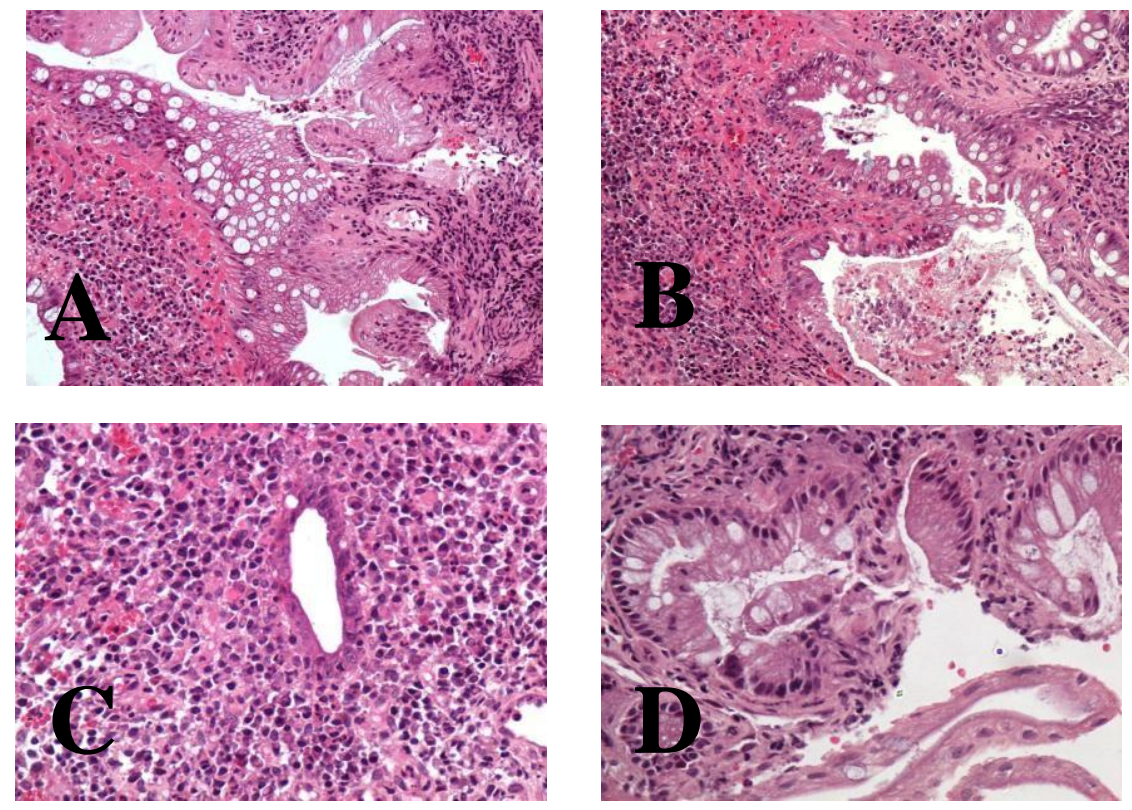

Fig.(2):- (A) UC showing crypt distortion, and irregular villous surface., (B) UC showing crypt abscesses within a disrorted crypts, (C) UC showing crypt atrophy together with a dense chronic inflammatory infiltrate of the lamina propria, (D) UC showing low grade dysplasia with distorted architecture, increased N/C ratio, and decreased mucus production.
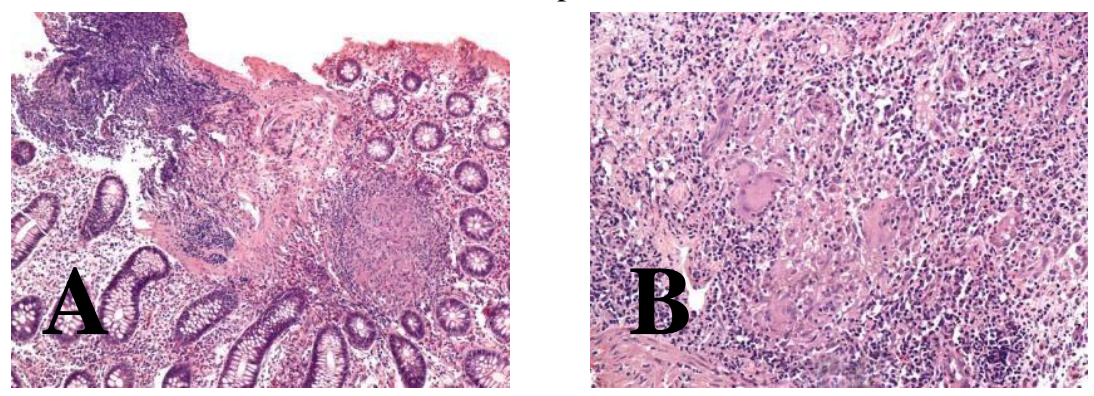

Fig.(3):- (A) CD showing dense inflammation and the presence of granuloma, (B) Higher magnification showing typical granuloma formed of epithelioid cells, giant cells, and many lymphocytes.

\section{Discussion:-}

Although Crohn's disease and ulcerative colitis have been fully described in several studies, continue to present a problem in the differential diagnosis from the clinical as well as the histopathological point of view. The two diseases share many clinical and histopathological features and the discriminating characteristics are often ill defined. 
Our purpose in this study was to study the characteristic clinicopathological features which help in the differential diagnosis between UC and CD, and assess the disease activity in ulcerative colitis. Because a better knowledge of the evolution of the microscopic features would be helpful in the treatment for patients with IBD according to the diagnosis and disease extent, severity and activity. Knowledge of disease activity could also be important for the prediction of relapse and for follow up of patients with dysplasia periodically to avoid development ofcancer.

In the recent years, Time trend analysis showed statistically significant increases in the incident of IBD over time. In 2012, the prevalence of IBD in Asia and Middle East was 6.3 per 100,000 person-years. Molodecky et al., (2012). (16).

The current work was carried out on 41 adequate endoscopic biopsies and 9 surgical resection specimens. The cases were categorized as follows: 27 ulcerative colitis and 23 Crohn's disease.

In this study, it was found that the mean age of patients of ulcerative colitis was 38.8 , with slight female's predominance (56\%). This was in agreement with Cotran, (1999) who recorded that the onset of disease peaks between the ages of $20-25$ years, and women are affected more often than men are. Similar findings were also obtained by Seldenrijk, (1991) and Berre et al., (1995) who found that the mean age for UC was 40 years and 39 years respectively, but the opposite was detected for sex predominance as they found that men were affected more often than women. $(17,18,19)$.

As regard the complaint, the main complaints were diarrhea and abdominal pain ( $76 \%$ both), followed by rectal bleeding (56\%), and only $8 \%$ of patients complained of extraintestinal symptoms such as arthritis. These findings were in accordance with Seldenrijk, (1991). (18).

Concerning colonoscopic examination 56\% of cases showed ulceration, pseudopolyposis was detected in $38 \%$ of cases, while edema and hyperemia was found in $32 \%$ of cases. Barium enema examination revealed ulcers in $48 \%$ of cases, spasm (12\%), narrowing (35\%), and pseudopolyposis $(20 \%)$, while it was free in $4 \%$ of cases. These findings were supported by Langmead, (2002). (20).

In this work, 27 cases were diagnosed as ulcerative colitis out of them, 1(3.7\%) case was diagnosed as grade $0.3,2$ (7.4\%) cases as grade 1.3, $2(18.5 \%)$ cases as grade 2A.2, $2(7.4 \%)$ as grade 2B.2, 1(3.7\%) case as grade 2B.3, 2

(7.4\%) cases as grade $3.1,4(14.8 \%)$ cases as grade $3.3,3(11.1 \%)$ cases as grade $4.1,4(14.8 \%)$ cases as grade 4.3 , $3(11.1 \%)$ as grade $5.4,2(7.4 \%)$ cases with low grade dysplasia and $1(3.7 \%)$ case with high grade dysplasia.

Nearly similar results were obtained reported before in the literature $(11,12,21,22)$. Tsang, (1999) reported that there are major and minor criteria for the diagnosis of UC, the major ones were: patchy mucosal inflammatory infiltrate, basal plasmacytosis, neutrophils overrunning mucosa, crypt abscesses, cryptitis, crypt distortion and villiform surface, while the minor criteria were: decreased goblet cells, and Paneth cell metaplasia.(23).

Theodossi, et al. (1994), suggested that a decrease in mucous content, an irregular or a villous surface, crypt distortion, diffuse crypt abscesses, and cryptitis are predictive of ulcerative colitis rather than of Crohn's disease. (24).

In the current work, Bcl-2 staining of UC mucosal $\mathrm{T}$ cells showed mild expression of the Bcl-2 protein and this indicated that UC mucosa contains few numbers of Bcl-2+ cells. These findings were also detected by Kenji Ina et al., (1999). (25).

In the current study two cases showed low-grade dysplasia, and one case showed high-grade dysplasia, the histopathological features seen in low-grade dysplasia were decreased intracellular mucin, nuclear hyperchromasia and pleomorphism, irregular nuclear membranes, and loss of nuclear polarity. In high-grade dysplasia, the following features were seen: enlarged hyperchromatic nuclei, marked nuclear stratification with crowding, marked loss of nuclear polarity, marked architectural distortion, and marked hyperchromasia and pleomorphism. These figures were consistent with those obtained by Neumann et al., (2011).(26).

In Crohn's disease, the age of predilection was between 22-64 years with a mean of 43.8. Females were affected more than males (60\%). This was in agreement with Cotran, (1999) who recorded that the peak age of detection are the teens and twenties with a minor peak in the $5^{\text {th }}$ and $6^{\text {th }}$ decades, and females were affected slightly more than males.(17). 
Clinically most cases were represented by diarrhea (65\%), abdominal pain (70\%), and 20\% of patients complained of anal complications such as fissures. These findings were consistent with those of Robert, (1998).(27).

Endoscopically, all cases revealed rectal sparing; discrete mucosal ulceration was detected in $45 \%$ of cases, $35 \%$ of cases showed pseudodiverticulosis, but skip lesions were detected in 55\% of cases only. These findings were coincided with Quinn, et al., (1994). (28).

Histopathological examination revealed transmural inflammation in all cases, preserved crypt architecture, which was seen in $80 \%$ of cases, microgranuloma in $40 \%$ of cases, dilatation of lymphatic channels (35\%), maintenance of goblet cell population (80\%), ulcereration (65\%), isolated giant cells (35\%), excess of histiocytes (65\%), Focal crypt abscess (25\%), and lymphoid aggregates in $65 \%$ of cases. While the distinctive feature of CD non caseatingepitheloid granuloma could be detected in only $45 \%$ of cases. These results were in keeping with the previously published data $(6,8,10,12,19,26,29,30)$. On the other hand, these findings were not compatible with those of Tsang (1999), who recorded that crypt abscesses and cryptitis are major features of CD, while crypt distortion (usually milder than in UC) is a minor feature.(23).

Examination of sections stained immunohistochemically for the antiapoptotic Bcl-2 protein revealed moderate expression of the Bcl-2 protein and this indicate that in $\mathrm{CD}$ there is a defect in apoptosis which in turn lead to prolongation of T cell survival and contribute to their accumulation in the mucosa. Kenji Ina et al., (1999) obtained nearly similar results and concluded that the number of Bcl-2+ cells can be taken as a discriminating factor between $\mathrm{UC}$ and CD. (25).

Tontini, (2015) reported that the gold standard for microscopic differentiation between Crohn's disease and ulcerative colitis should be established by collecting two biopsies from at least five sites along the colon.(31)

In conclusion, this study using multiple parameters: histological features and Bcl-2 expression as studied by immunohistochemical stain, was a trial to gain an accurate differentiation between Crohn's disease and ulcerative colitis. According to these parameters, we were able to distinguish between 50 cases of IBD, 27 cases of UC with different grades, 1 case of UC with dysplasia and 23 cases of CD.

\section{References:-}

1. Shepherd, N. (1991). Pathological mimics of chronic inflammatory bowel disease. Journal Of Clinical Pathology, 44(9), 726-733.Web

2. Cobrin, G., \& Abreu, M. (2005). Defects in mucosal immunity leading to Crohn's disease. Immunol Rev, 206(1),277-295.web

3. Targan, S., \& Karp, L. (2005). Defects in mucosal immunity leading to ulcerative colitis. Immunol Rev, 206(1), 296-305.Web

4. Jin J. Inflammatory Bowel Disease. JAMA. 2014;311(19):2034. doi:10.1001/jama.2014.1664.

5. Ha, F., \& Khalil, H. (2015). Crohn's disease: a clinical update. Therapeutic Advances In Gastroenterology, 8(6), 352-359.Web

6. James, S. (2014). Identification of pathologic features associated with "ulcerative colitis-like" Crohn'sdisease. World Journal of Gastroenterology, 20(36), p.13139.Web

7. Torpy JM, LynmC, GolubRM. Ulcerative Colitis. JAMA. 2012;307(1):104.doi:10.1001/jama.2011.1889.

8. Differentiating Ulcerative Colitis from Crohn Disease in Children and Young Adults: Report of a Working Group of the North American Society for Pediatric Gastroenterology, Hepatology, and Nutrition and the Crohn's and Colitis Foundation of America. (2007). Journal of Pediatric Gastroenterology and Nutrition, 44(5), pp.653-674.Web

9. Reid, M., \&Stehr, W.(2010) The Mont Reid surgicalhandbook.

10. Stange, E. (2006). European evidence based consensus on the diagnosis and management of Crohn's disease: definitions and diagnosis. Gut, 55(suppl_1), i1-i15.Web

11. Stange, E., Travis, S., Vermeire, S., Reinisch, W., Geboes, K., \&Barakauskiene, A. et al. (2008). European evidence-based Consensus on the diagnosis and management of ulcerative colitis: Definitions and diagnosis. Journal Of Crohn's And Colitis, 2(1), 1-23.Web

12. Kim, D., Lee, K., Lee, J., Chung, Y., Sung, H., Paik, C., Chung, W., Jung, J. and Choi, H. (2016). Correlation between Histological Activity and Endoscopic, Clinical, and Serologic Activities in Patients with Ulcerative Colitis. Gastroenterology Research and Practice, 2016,pp.1-7 
13. Garud, S., \& Peppercorn, M. (2009). Review: Ulcerative colitis: current treatment strategies and future prospects. Therapeutic Advances In Gastroenterology, 2(2), 99-108.Web

14. Althumairi, A. (2016). Inflammatory bowel disease associated neoplasia: A surgeon's perspective. World Journal Of Gastroenterology,22(3),961.Web

15. Mark A Peppercorn, MD,Sunanda V Kane, MD, MSPH Clinical manifestations, diagnosis and prognosis of Crohn disease in adults, Clinical manifestations, diagnosis and prognosis of Ulcerative colitis in adults. Apr 22, 2014

16. Molodecky, N., Soon, I., Rabi, D., Ghali, W., Ferris, M., \&Chernoff, G. et al. (2012). Increasing Incidence and Prevalence of the Inflammatory Bowel Diseases With Time, Based on Systematic Review. Gastroenterology, 142(1), 46-54.e42.Web

17. Cotran RS, Kumar V, Collins T (1999): Idiopathic inflammatory bowel disease. In Robbins Pathologic Basis of Disease. 6th ed W.B. Saunders Company Philadelphia, London, Toronto. Montreal, Sydney and Tokyo; p. 815820.

18. Seldenrijk, C., Morson, B., Meuwissen, S., Schipper, N., Lindeman, J., \& Meijer, C. (1991). Histopathological evaluation of colonic mucosal biopsy specimens in chronic inflammatory bowel disease: diagnostic implications. Gut, 32(12), 1514-1520.Web

19. Le Berre, N., Heresbach, D., Kerbaol, M., Caulet, S., Bretagne, J., \& Chaperon, J. et al. (1995). Histological discrimination of idiopathic inflammatory bowel disease from other types of colitis. Journal Of Clinical Pathology, 48(8), 749-753.web

20. Langmead, L., \&Rampton, D. (2002). Plain abdominal radiographic features are not reliable markers of disease extent in active ulcerative colitis. The American Journal Of Gastroenterology, 97(2), 354-359. Web

21. Domizio, P. (1994). Pathology of chronic inflammatory bowel disease in children. Baillière's Clinical Gastroenterology, 8(1), 35-63.Web

22. Geboes, K. (2001). Pathology of inflammatory bowel diseases (IBD): variability with time and treatment. Colorect Dis, 3(1), 2-12.web

23. Tsang, P., \& Rotterdam, H. (1999). Biopsy Diagnosis of Colitis. The American Journal Of Surgical Pathology, 23(4), 423-430. Web

24. Theodossi, A., Spiegelhalter, D., Jass, J., Firth, J., Dixon, M., \& Leader, M. et al. (1994). Observer variation and discriminatory value of biopsy features in inflammatory bowel disease. Gut, 35(7), 961-968. Web

25. Kenji Ina, JugohItoh, Kouhei Fukushima, KazouKusugami, Takeo Yamaguchi, Kazuhiro Kyokane, Thomas S. McCormick, Eduardo G. Lapetina, et al. (1999): Resistance of Crohn's disease T cells to multiple apoptotic signals is associated with a Bcl-2/Bax mucosal imbalance. The journal of immunology 163: 1081-1090

26. Neumann, H., Vieth, M., Langner, C., Neurath, M. F., \&Mudter, J. (2011). Cancer risk in IBD: How to diagnose and how to manage DALM and ALM. World Journal of Gastroenterology: WJG, 17(27), web

27. Robert MG, (1998): IBD: UC and CD. In: Harrison's Principles of Intrnal Medicine, 14ed. P:1633.

28. Quinn PG, Binion DG, Connors PJ (1994): the role of endocopy in inflammatory bowel disease. Med Clin N Am 78: 1331-52.

29. Martin Farmer, F.R.C.S., Robert E. Petras, Louise E. Hunt, F.R.C.S., Janine E. Janosky, Susan Galandiuk (2000): the importance of diagnostic accuracy in colonic inflammatory bowel disease. Am J Gastroenterol 95: 3184- 3188 .

30. Cross, S S, and R F Harrison. "Discriminant Histological Features In The Diagnosis Of Chronic Idiopathic Inflammatory Bowel Disease: Analysis Of A Large Dataset By A Novel Data Visualisation Technique". Journal of Clinical Pathology 55.1 (2002): 51-57.Web.

31. Tontini, GianEugenio. "Differential Diagnosis In Inflammatory Bowel Disease Colitis: State Of The Art And Future Perspectives". World Journal of Gastroenterology 21.1 (2015): 21.Web. 